Korean J. Math. 21 (2013), No. 2, pp. 151-159

http://dx.doi.org/10.11568/kjm.2013.21.2.151

\title{
THE $\lambda$-NUMBER OF THE CARTESIAN PRODUCT OF A COMPLETE GRAPH AND A CYCLE
}

\author{
Byeong Moon Kim, Byung Chul Song and Yoomi Rho*
}

\begin{abstract}
An $L(j, k)$-labeling of a graph $G$ is a vertex labeling such that the difference of the labels of any adjacent vertices is at least $j$ and that of any vertices of distance two is at least $k$ for given $j$ and $k$. The minimum span of all $L(2,1)$-labelings of $G$ is called the $\lambda$-number of $G$ and is denoted by $\lambda(G)$.

In this paper, we find a lower bound of the $\lambda$-number of the Cartesian product $K_{m} \square C_{n}$ of the complete graph $K_{m}$ of order $m$ and the cycle $C_{n}$ of order $n$. In fact, we show that when $n \geq 3$, $\lambda\left(K_{4} \square C_{n}\right) \geq 7$ and the equality holds if and only if $n$ is a multiple of 8 . Moreover when $m \geq 5, \lambda\left(K_{m} \square C_{n}\right) \geq 2 m-1$ and the equality holds if and only if $n$ is even.
\end{abstract}

\section{Introduction}

In a wireless communication network, a channel assignment problem(CAP) involves assigning channels which are represented by nonnegative integers, to the radio transmitters in the network. There must be some restrictions to minimize interference between the channels assigned to the transmitters that are close or very close. The restrictions would

Received March 13, 2013. Revised May 14, 2013. Accepted May 20, 2013.

2010 Mathematics Subject Classification: 05C78(05C38).

Key words and phrases: $L(2,1)$-labeling, $\lambda$-number, Cartesian product, complete graph, cycle.

This work is supported by the University of Incheon research grant in 2008-2009.

*Corresponding author.

(c) The Kangwon-Kyungki Mathematical Society, 2013.

This is an Open Access article distributed under the terms of the Creative commons Attribution Non-Commercial License (http://creativecommons.org/licenses/by -nc/3.0/) which permits unrestricted non-commercial use, distribution and reproduction in any medium, provided the original work is properly cited. 
be as follows: For a pair of positive integers $j, k(j \geq k)$, we assign channels with difference at least $j$ to transmitters which are very close and at least $k$ to those which are close.

CAP is first placed in a graph theoretic context in a more generalized form by Hale[8] in the following way. Vertices of a graph represent the transmitters and two vertices are adjacent if the corresponding transmitters are very close.

A distance two labeling or $L(j, k)$-labeling of a graph $G=\left(V_{G}, E_{G}\right)$ is a function $f: V_{G} \rightarrow[0, \infty)$ such that

$$
|f(u)-f(v)| \geq \begin{cases}j & \text { if } \operatorname{dist}_{G}(u, v)=1 \\ k & \text { if } \operatorname{dist}_{G}(u, v)=2 .\end{cases}
$$

We call the elements of the image of $f$ the labels and the difference between the maximum and the minimum of the labels assigned by $f$ the span of $f$. Usually we assume that the minimum label zero, and hence we regard the maximum label of $f$ as the span of $f$. Furthermore the minimum span over all labelings is called the $\lambda_{k}^{j}$-number of $G$ and is denoted by $\lambda_{k}^{j}(G)$. Griggs and Yeh[6] proposed a problem of determining $\lambda_{k}^{j}(G)$, especially $\lambda_{1}^{2}(G)$, and proved that for a graph $G$ of maximum degree $\Delta, \lambda_{1}^{2}(G) \leq \Delta^{2}+2 \Delta$. They also conjectured that $\lambda_{1}^{2}(G) \leq \Delta^{2}$. After their work, $\lambda_{1}^{2}(G)$ is called the $\lambda$-number of $G$ and denoted by $\lambda(G)$. The $\lambda$-number of some classes of graphs have been studied in many literatures $[1,13,12,4,10]$. See $[2,3,5]$ for extensive surveys.

For two graphs $G$ and $H$, the Cartesian product of $G$ and $H$, which is denoted by $G \square H$ is the graph with vertex set $V_{G} \times V_{H}$ where two vertices $(x, y)$ and $\left(x^{\prime}, y^{\prime}\right)$ are adjacent if (i) $x=x^{\prime}$ and $\left(y, y^{\prime}\right) \in E_{H}$, or (ii) $y=y^{\prime}$ and $\left(x, x^{\prime}\right) \in E_{G}$.

There are results on the $\lambda_{k}^{j}$-number of the Cartesian product of various simple graphs $[19,7,11,18,16,9,15,14]$.

In this paper, we find a lower bound of the $\lambda$-number of the Cartesian product $K_{m} \square C_{n}$ of the complete graph $K_{m}$ of order $m$ and the cycle $C_{n}$ of order $n$. In fact, we show that when $n \geq 3, \lambda\left(K_{4} \square C_{n}\right) \geq 7$ and the equality holds if and only if $n$ is a multiple of 8 . We also show that when $m \geq 5, \lambda\left(K_{m} \square C_{n}\right) \geq 2 m-1$ and the equality holds if and only if $n$ is even. 


\section{The $\lambda$-number of $K_{m} \square C_{n}$}

Let $G=K_{m} \square C_{n}=(V, E)$ be the Cartesian product of the complete graph $K_{m}$ on $m$ vertices and the cycle $C_{n}$ on $n$ vertices. See Figure 1 for $G=K_{5} \square C_{6}$ as an example.

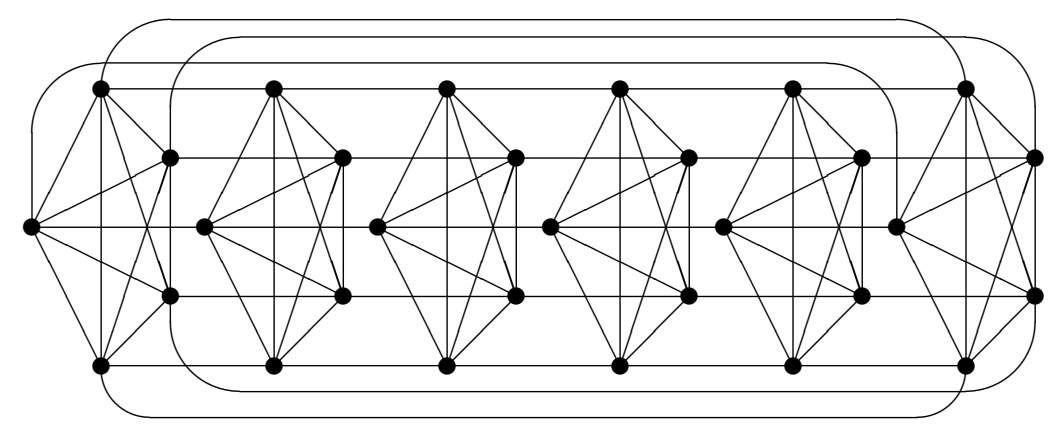

Figure 1. $K_{5} \square C_{6}$

For each $x \in \mathbb{Z}$ and $M \in \mathbb{Z}^{+}$, the residue of $x$ modulo $M$ is denoted by $x(\bmod M)$. Note that a function $f$ from $V$ to $[0, N]$ for some positive integer $N$ is an $L(2,1)$-labeling of $G$ if and only if it satisfies

(i) $\left|\mathrm{f}(\mathrm{i}, \mathrm{j})-\mathrm{f}\left(\mathrm{i}^{\prime}, \mathrm{j}^{\prime}\right)\right| \geq 2$ if either $i \neq i^{\prime}$ and $j=j^{\prime}$,

$$
\text { or } i=i^{\prime} \text { and } j=j^{\prime} \pm 1 \quad(\bmod n) \text {, }
$$

(ii) $\left|\mathrm{f}(\mathrm{i}, \mathrm{j})-\mathrm{f}\left(\mathrm{i}^{\prime}, \mathrm{j}^{\prime}\right)\right| \geq 1$ if either $i \neq i^{\prime}$ and $j=j^{\prime} \pm 1$, or $i=i^{\prime}$ and $j=j^{\prime} \pm 2 \quad(\bmod n)$.

The following lemma is easily proved.

Lemma 1. Let $G=K_{m} \square C_{n}=(V, E)$ and $f: V \rightarrow[0, N]$ be a function. Extend $f$ to all $i \in[0, m-1]$ and $j \in \mathbb{Z}$ by $f(i, j)=f(i, \tilde{j})$ where $j=\tilde{j}(\bmod n)$. Then $f$ is an $L(2,1)$-labeling of $G$ if and only if the extended $f$ satisfies

$$
\begin{gathered}
(\mathrm{i})\left|\mathrm{f}(\mathrm{i}, \mathrm{j})-\mathrm{f}\left(\mathrm{i}^{\prime}, \mathrm{j}^{\prime}\right)\right| \geq 2 \text { if either } i \neq i^{\prime} \text { and } j=j^{\prime} \quad(\bmod n), \\
\text { or } i=i^{\prime} \text { and } j=j^{\prime} \pm 1 \quad(\bmod n), \\
(\text { ii })\left|\mathrm{f}(\mathrm{i}, \mathrm{j})-\mathrm{f}\left(\mathrm{i}^{\prime}, \mathrm{j}^{\prime}\right)\right| \geq 1 \\
\text { if either } i \neq i^{\prime} \text { and } j=j^{\prime} \pm 1 \quad(\bmod n), \\
\text { or } i=i^{\prime} \text { and } j=j^{\prime} \pm 2 \quad(\bmod n) .
\end{gathered}
$$


Schwartz and Troxell[17] proved that

$$
\lambda\left(K_{3} \square C_{n}\right)= \begin{cases}7, & \text { if } n \text { is even with } n \neq 4,10, \\ 8, & \text { otherwise. }\end{cases}
$$

Theorem 2. For $m \geq 4$, we have the following lower bound of the $\lambda$-number of $K_{m} \square C_{n}$ which are extreme for some $n$.

(i) For each $n \geq 3, \lambda\left(K_{4} \square C_{n}\right) \geq 7$ and the equality holds if and only if $n$ is a multiple of 8 .

(ii) For each $m \geq 5$ and $n \geq 3, \lambda\left(K_{m} \square C_{n}\right) \geq 2 m-1$ and the equality holds if and only if $n$ is even.

Proof. Throughout this proof, let $G=K_{m} \square C_{n}=(V, E)$.

(i) Let $f: V \rightarrow[0, N]$ be an $L(2,1)$-labeling of $G$. We consider the extension of $f$ defined on $[0, m-1] \times \mathbb{Z}$ in Lemma 1 . For each nonnegative integer $j$, we define $V_{j}=\{(i, j) \mid 0 \leq i \leq 3\}$. Since for each $j$ any two elements of $V_{j} \cup V_{j+1}$ are of distance at most two, $f\left(V_{j} \cup V_{j+1}\right)=$ $\left\{f(v) \mid v \in V_{j} \cup V_{j+1}\right\}$ has eight elements. Thus $8 \leq N+1$ so that $N \geq 7$. It follows that $\lambda(G) \geq 7$.

We show that the equality holds if and only if $n$ is a multiple of 8 . Assume $N=7$. For each $j$, since $f\left(V_{j} \cup V_{j+1}\right)$ has eight elements, $f\left(V_{j} \cup\right.$ $\left.V_{j+1}\right)=[0,7]$. We may assume that $0 \in f\left(V_{0}\right)$ and hence $f\left(V_{1}\right) \subset[1,7]$. Considering that any two labels of distinct elements of $V_{1}$ are apart by at least two, we have $f\left(V_{1}\right)=\{1,3,5,7\}$. Therefore $f\left(V_{0}\right)=\{0,2,4,6\}$. Similarly,

$$
f\left(V_{j}\right)= \begin{cases}\{0,2,4,6\}, & \text { if } j \text { is even, } \\ \{1,3,5,7\}, & \text { if } j \text { is odd. }\end{cases}
$$

We may assume that $f(i, 0)=2 i$ for $0 \leq i \leq 3$. There is $i_{1}$ such that $f\left(i_{1}, 1\right)=1$. Since $\left|f\left(i_{1}, 0\right)-f\left(i_{1}, 1\right)\right|=\left|2 i_{1}-1\right| \geq 2, i_{1}$ is 2 or 3 .

If $i_{1}=2$, then we want to prove that

$$
f(i, j)=2 i+5 j \quad(\bmod 8)
$$

for all $i, j$. Since $f(3,1) \in\{1,3,5,7\},|f(3,1)-f(3,0)|=|f(3,1)-6| \geq$ 2 and $f(3,1) \neq f(2,1)=1$, we have $f(3,1)=3$. Since $f(2,2) \in$ $\{0,2,4,6\},|f(2,2)-f(2,1)|=|f(2,2)-1| \geq 2$ and $f(2,2) \neq f(2,0)=4$, we have $f(2,2)=6$. Since $|f(3,2)-f(3,1)|=|f(3,2)-3| \geq 2$ and $f(3,2) \neq f(3,0)=6$, we have $f(3,2)=0$. Since $f(1,2) \in\{0,2,4,6\}$ and $f(1,2)$ is different from $f(1,0)=2, f(2,2)=6, f(3,2)=0$, we have $f(1,2)=4$. Thus $f(0,2)=2$. Since $f(1,1) \in\{5,7\}$ and $\mid f(1,1)-$ $f(1,2)|=| f(1,1)-4 \mid \geq 2$, we have $f(1,1)=7$. Thus $f(0,1)=5$. Note 
that $f(i, 1)=2 i+5(\bmod 8)$ and $f(i, 2)=2 i+2(\bmod 8)=2 i+2 \cdot 5$ $(\bmod 8)$. We just proved that if equation $(1)$ holds for $j=0$ and $(i, j)=$ $(2,1)$, then equation (1) holds for $j=1,2$ and $(i, j)=(1,3)$. By the same method, we can prove that for any $t$, if equation (1) holds for $j=2 t$ and $(i, j)=(s, 2 t+1)$ where $s=2-t(\bmod 4)$, then equation $(1)$ holds for $j=2 t+1,2 t+2$ and $(i, j)=\left(s^{\prime}, 2 t+3\right)$ where $s^{\prime}=2-(t+1)$ (mod 4$)$. Thus by induction, equation $(1)$ is true for all $j$. Since

$$
0=f(0,0)=f(0, n)=5 n \quad(\bmod 8),
$$

$n$ is a multiple of 8 .

If $i_{1}=3$, then by a similar method as above, we can prove

$$
f(i, j)=2 i+3 j \quad(\bmod 8)
$$

holds for all $i, j$. Since

$$
0=f(0,0)=f(0, n)=3 n \quad(\bmod 8),
$$

$n$ is a multiple of 8 .

Thus for any $i_{1}, n$ is a multiple of 8 . It follows that $\lambda(G)=7$ only if $n$ is a multiple of 8 .

Conversely assume $n$ is a multiple of 8 . Then since

$$
f(i, j)=2 i+5 j \quad(\bmod 8)=2 i+5 j+5 n \quad(\bmod 8)=f(i, j+n)
$$

and

$$
f(i, j)=2 i+3 j \quad(\bmod 8)=2 i+3 j+3 n \quad(\bmod 8)=f(i, j+n),
$$

two labelings defined by equation (1) and equation (2) are well-defined. These labelings are shown in Table 1 . Thus $\lambda(G)=7$ when $n$ is a multiple of 8 .

(ii) Let $f: V \rightarrow[0, N]$ be an $L(2,1)$-labeling of $G$. We consider the extension of $f$ defined on $[0, m-1] \times \mathbb{Z}$ in Lemma 1 . For each $j \in[0, n-1]$, define $V_{j}=\{(i, j) \mid 0 \leq i \leq m-1\}$. Since for each $j$, any two elements of $V_{j} \cup V_{j+1}$ are of distance at most two, $f\left(V_{j} \cup V_{j+1}\right)$ has $2 m$ elements. Thus $2 m \leq N+1$ so that $N \geq 2 m-1$. It follows that $\lambda(G) \geq 2 m-1$.

We show that the equality holds if and only if $n$ is even. Assume $N=$ $2 m-1$. Since for each $j, f\left(V_{j} \cup V_{j+1}\right)$ has $2 m$ elements, $f\left(V_{j} \cup V_{j+1}\right)=$ $[0,2 m-1]$. We may assume that $0 \in f\left(V_{j}\right)$. Then $f\left(V_{1}\right) \subset[1,2 m-1]$ and hence we have $f\left(V_{1}\right)=\{1,3, \cdots, 2 m-1\}$ considering that any two labels 
of distinct elements of $V_{1}$ are apart by at least two. Since $f\left(V_{0} \cup V_{1}\right)=$ $f\left(V_{0}\right) \cup f\left(V_{1}\right)=[0,2 m-1]$, we have $f\left(V_{0}\right)=\{0,2, \cdots, 2 m-2\}$. Similarly,

$$
f\left(V_{j}\right)= \begin{cases}\{0,2, \cdots, 2 m-2\}, & \text { if } j \text { is even, } \\ \{1,3, \cdots, 2 m-1\}, & \text { if } j \text { is odd. }\end{cases}
$$

Since $V_{n}=V_{0}, n$ is even. Thus $\lambda(G)=2 m-1$ only if $n$ is even.

Conversely assume $n$ is even. If $m=5$, then there is an $L(2,1)$ labeling

$$
f_{1}(i, j)=2 i+a_{j} \quad(\bmod 2 m)
$$

for $\left(a_{0}, a_{1}, a_{2}, a_{3}\right)=(0,3,8,5)$ of $K_{5} \square C_{4}$ and an $L(2,1)$-labeling

$$
f_{2}(i, j)=2 i+3 j \quad(\bmod 2 m)
$$

of $K_{5} \square C_{6}$, which are shown in Table 2. Since $n$ is even and at least 4, $n=4 x+6 y$ for some non-negative integer $x, y$. Therefore

$$
f(i, j)= \begin{cases}f_{1}(i, j), & \text { if } 0 \leq j<4 x \\ f_{2}(i, j-4 x), & \text { if } 4 x \leq j \leq n-1 .\end{cases}
$$

is an $L(2,1)$-labeling of $G=K_{5} \square C_{n}$ with its span 9. Thus $\lambda(G)=9$.

If $m \geq 6$, then

$$
f_{3}(i, j)= \begin{cases}2 i+j \quad(\bmod 2 m), & \text { if } j \text { is even } \\ 2 i+j+5 \quad(\bmod 2 m), & \text { if } j \text { is odd. }\end{cases}
$$

is an $L(2,1)$-labeling of $K_{m} \square C_{4}$ and also an $L(2,1)$-labeling of $K_{m} \square C_{6}$. They are shown in Table 3. Since $n$ is even and at least $4, n=4 x+6 y$ for some non-negative integer $x, y$. Therefore

$$
f(i, j)= \begin{cases}f_{3}(i, j), & \text { if } 0 \leq j<4 x \\ f_{3}(i, j-4 x), & \text { if } 4 x \leq j \leq n-1 .\end{cases}
$$

is an $L(2,1)$-labeling of $G=K_{m} \square C_{n}$ with its span $2 m-1$. Thus $\lambda(G)=$ $2 m-1$.

\begin{tabular}{|c|cccccccc|}
\hline$i \backslash^{j}$ & 0 & 1 & 2 & 3 & 4 & 5 & 6 & 7 \\
\hline 0 & 0 & 5 & 2 & 7 & 4 & 1 & 6 & 3 \\
1 & 2 & 7 & 4 & 1 & 6 & 3 & 0 & 5 \\
2 & 4 & 1 & 6 & 3 & 0 & 5 & 2 & 7 \\
3 & 6 & 3 & 0 & 5 & 2 & 7 & 4 & 1 \\
\hline
\end{tabular}

\begin{tabular}{|c|cccccccc|}
\hline$i \searrow^{j}$ & 0 & 1 & 2 & 3 & 4 & 5 & 6 & 7 \\
\hline 0 & 0 & 3 & 6 & 1 & 4 & 7 & 2 & 5 \\
1 & 2 & 5 & 0 & 3 & 6 & 1 & 4 & 7 \\
2 & 4 & 7 & 2 & 5 & 0 & 3 & 6 & 1 \\
3 & 6 & 1 & 4 & 7 & 2 & 5 & 0 & 3 \\
\hline
\end{tabular}

TABLE 1. Two $L(2,1)$-labelings of $K_{4} \square C_{8}$ 


\begin{tabular}{|c|cccc}
\hline$i \backslash^{j}$ & 0 & 1 & 2 & 3 \\
\hline 0 & 0 & 3 & 8 & 5 \\
1 & 2 & 5 & 0 & 7 \\
2 & 4 & 7 & 2 & 9 \\
3 & 6 & 9 & 4 & 1 \\
4 & 8 & 1 & 6 & 3
\end{tabular}$\quad$\begin{tabular}{|c|cccccc|}
$i^{\prime} \backslash^{j}$ & 0 & 1 & 2 & 3 & 4 & 5 \\
0 & 0 & 3 & 6 & 9 & 2 & 5 \\
1 & 2 & 5 & 8 & 1 & 4 & 7 \\
2 & 4 & 7 & 0 & 3 & 6 & 9 \\
3 & 6 & 9 & 2 & 5 & 8 & 1 \\
4 & 8 & 1 & 4 & 7 & 0 & 3 \\
\hline
\end{tabular}

TABLE 2. $L(2,1)$-labelings of $K_{5} \square C_{4}$ and $K_{5} \square C_{6}$

\begin{tabular}{|c|cccc|}
\hline$i \searrow^{j}$ & 0 & 1 & 2 & 3 \\
\hline 0 & 0 & 5 & 2 & 7 \\
1 & 2 & 7 & 4 & 9 \\
2 & 4 & 9 & 6 & 11 \\
$\vdots$ & $\vdots$ & $\vdots$ & $\vdots$ & $\vdots$ \\
$m-1$ & $2 m-2$ & 3 & 0 & 5 \\
\hline
\end{tabular}

\begin{tabular}{|c|c|c|c|c|c|c|c|}
\hline$i \searrow^{j}$ & 0 & 1 & 2 & 3 & 4 & & 5 \\
\hline 0 & 0 & 5 & 2 & 7 & 4 & & 9 \\
\hline 1 & 2 & 7 & 4 & 9 & 6 & & 11 \\
\hline 2 & 4 & 9 & 6 & 11 & 8 & $\begin{array}{l}13, \\
1,\end{array}$ & $\begin{array}{l}\text { if } m \geq 7, \\
\text { if } m=6 \text {. }\end{array}$ \\
\hline$\vdots$ & $\vdots$ & : & $\vdots$ & : & : & & $\vdots$ \\
\hline$m-1$ & $2 m-2$ & 3 & 0 & 5 & 2 & & 7 \\
\hline
\end{tabular}

TABLE 3. $L(2,1)$-labelings of $K_{m} \square C_{4}$ and $K_{m} \square C_{6}$ for $m \geq 6$

\section{References}

[1] S.S. Adams, J. Cass and D.S. Troxell, An extension of the channelassignment problem: L(2,1)-labelings of generalized Petersen graphs, IEEE Trans. Circ. Sys. I-Regular Papers, 53 (2006), 1101-1107.

[2] T. Calamoneri, The $L(h, k)$-labeling problem: A survey and annotated biblography, Computer Journal, 49 (2006), 585-608. 
[3] T. Calamoneri, The $L(h, k)$-labeling problem: An updated survey and annotated biblography, Computer Journal, 54 (2011), 1344-1371.

[4] R. Erman, S. Jurečič, D. Kral, K. Stopar and N. Stopar, Optimal real number graph labellings of a subfamily of Kneser graphs, (English summary), SIAM J. Disc. Math. 23 (2009), 1372-1381.

[5] J.R. Griggs and X.T. Jin, Recent progress in mathematics and engineering on optimal graph labelings with distance conditions, J. of Comb. Optim. 14 (2007), 249-257.

[6] J.R. Griggs and R.K. Yeh, Labeling graphs with acondition at distance two, SIAM J. Disc. Math 5 (1992), 586-595.

[7] J.P. Georges, D.W. Mauro and M. I. Stein, Labeling products of complete graphs with a condition at a distance two, SIAM J. Disc. Math. 14 (2000), 2-35.

[8] W.K. Hale, Frequency assignment: theory and application, Proc. IEEE 68 (1980), 1497-1514.

[9] E. Haque and P. K. Jha, $L(j, k)$-labelings of Kronecker products of complete graphs, IEEE Trans. on Cir. and Sys. II 55 (2008), 70-73.

[10] F. Havet, B. Reed and J. Sereni, Griggs and Yeh's Conjecture and L $(p, 1)-$ labelings, SIAM J. Disc. Math. 26 (2012), 145-168.

[11] P.K. Jha, S. Klavžar and A. Vesel, Optimal L(d,1)-labelings of certain direct products of cycles and Cartesian product of cycles, Disc. Appl. Math. 152 (2005), 257-265.

[12] J. Kang, L(2,1)-labeling of hamiltonian graphs with maximum degree 3, SIAM J. Disc. Math. 22 (2008), 213-230.

[13] S. Klavzăr and S. Špacapan, The $\Delta^{2}$-conjecture for $L(2,1)$-labelings is true for directed and strong products of graphs, IEEE Trans. Circuits Syst. II 53(2006), 274-277.

[14] B. Kim, B. Song and Y. Rho, Labeling products of complete graphs with a condition at a distance two, International J. Computer Mathematics, in press.

[15] W. Lin and P.C.B. Lam, Distance two labeling and direct products of graphs, Disc. Math. 308 (2008), 3805-3815.

[16] P. Lam, W. Lin and J. Wu, $L(j, k)$-labelings for the products of complete graphs, J. of Comb. Optim. 14 (2007), 219-227.

[17] C. Schwartz and D. Troxell, L $(2,1)$-labelings of Cartesian product of two cycles, Disc. Appl. Math. 154 (2006), 1522-1540.

[18] Z.D. Shao and R.K. Yeh, The L(2,1)-labeling and operations of graphs, IEEE Trans. Circ. Sys. I-Regular Papers 52 (2005), 668-671.

[19] M.A. Whittlesey, J.P. Georges and D.W. Mauro, On the $\lambda$-number of $Q_{n}$ and related graphs, SIAM J. Disc. Math. 8 (1995), 499-506. 
Department of Mathematics

Gangneung-Wonju National University

Korea

E-mail: kbm@gwnu.ac.kr

Department of Mathematics

Gangneung-Wonju National University

Korea

E-mail: bcsong@gwnu.ac.kr

Department of Mathematics

University of Incheon

Korea

E-mail: rho@incheon.ac.kr 